\title{
Estudos taxonômicos na família Eriocaulaceae em áreas prioritárias para conservação na Chapada Diamantina, no âmbito do PPBIO Semiárido
}

\author{
Mayra de Souza Pereira $^{1}$; Reyjane Patricia de Oliveira ${ }^{2}$ \\ 1. Bolsista PROBIC/UEFS, Graduanda em Ciências Biológicas, Universidade Estadual de Feira de Santana, e-mail: \\ mayra.pereira13@hotmail.com \\ 2. Orientador, Departamento de Ciências Biológicas, Universidade Estadual de Feira de Santana, e-mail: \\ rpatricia@uefs.br
}

PALAVRAS-CHAVE: Eriocaulaceae, Semiárido, Campo rupestre

\section{INTRODUÇÃO}

O Semiárido brasileiro inclui uma área de aproximadamente $900.000 \mathrm{~km}^{2}$, englobando 11 Estados, com uma população de mais de 18 milhões de habitantes (Rebouças, 1997; Malvezzi, 2001). Nessa região ocorrem diversos tipos de vegetações, que oferecem diferentes benefícios ambientais, econômicos e sociais, por isso muitos levantamentos são realizados envolvendo a flora da região, a fim de complementar ou acrescentar estudos sobre áreas ainda desconhecidas ou pouco conhecidas do ponto de vista florístico. A região da Chapada Diamantina está inserida nos limites do Semiárido e representa o maior complexo de montanhas do Brasil (Giulietti et al., 1997), sendo o campo rupestre o principal tipo de vegetação ocorrente na região. Eriocaulaceae é uma família facilmente caracterizada pelo hábito geralmente em roseta, de onde emergem os escapos, portando as inflorescências do tipo capítulo e um dos principais centros de diversidade da família encontra-se na Bahia, especificamente nas montanhas da Chapada. A diversidade dessa família ainda não é conhecida em sua totalidade, sendo assim, a proposta do trabalho foi dar continuidade aos estudos com Eriocaulaceae na Chapada Diamantina para elaboração de uma lista atualizada das espécies ocorrentes nessa região.

\section{METODOLOGIA}

Foi realizado um estudo sobre a caracterização da família para reconhecimento de seus indivíduos. Em seguida, foram feitas buscas sobre as espécies da família ocorrentes no Brasil, nordeste e Bahia através do site Flora do Brasil. O mesmo levantamento foi realizado através de consulta ao herbário virtual Species link e uma expedição foi realizada em 2016 na área de estudo, para complementação dos dados e reconhecimento do material em campo. As coletas seguiram os procedimentos padrões de prensagem, secagem e confecção de exsicatas (Mori et. Al., 1989). Em sequência, esses materiais foram incorporados ao banco de dados do HUEFS (Sigla segundo Thiers 2016). Os mesmos foram identificados através de chave de identificação elaborada Giulietti (2012) para os gêneros. A identificação das espécies, foi possível a partir dos trabalhos publicados pelos especialistas de cada gênero. Para elaboração da lista das espécies ocorrentes na área de estudos, os dados foram obtidos em literatura, etiquetas dos herbários e dados disponíveis nos sites Flora do Brasil e Species Link. Todas essas informações foram relacionadas e comparadas aos materiais identificados para montagem da lista. 


\section{RESULTADOS E DISCUSSÃO}

Através desse estudo foi possível confirmar a ocorrência de 72 espécies e seis gêneros de Eriocaulaceae para a região da Chapada Diamantina. Dentre os sete gêneros dessa família registrados na Bahia, apenas Tonina não foi encontrado na área, o qual é característico de ambientes aquáticos. O gênero com maior número de espécies na área foi Paepalanthus, com 37 espécies. Para o gênero Actinocephalus foi registrada a ocorrência de cinco espécies; Comanthera apresentou 12 espécies, Eriocaulon sete espécies, Leiothrix seis e Syngonanthus cinco. Essas espécies se encontram presentes em vegetações do tipo cerrado, campo rupestre, afloramentos rochosos e estão distribuídas ao longo de 12 cidades: Abaíra, Andaraí, Barra da Estiva, Itaetê, Morro do Chapéu, Palmeiras, Rio de Contas, Seabra, Ibicoara, Lençóis, Mucugê, Piatã. Os espécimes do material do PPBio e da coleção do HUEFS foram analisados a partir da chave de identificação de Giulietti (2012). Das 54 coletas realizadas pelo Projeto PPBio Semiárido foram identificadas 15 espécies: Leiothrix flavescens (Bong.) Ruhland, Paepalanthus pulchellus Herzog, Leiothrix hirsuta (Wikstr.) Ruhland, Paepalanthus erigeron Mart. ex Körn, Paepalanthus obtusifolius (Steud.) Körn, Paepalanthus carvalhoi Giul. \& E.B.Miranda, Paepalanthus regalis Mart, Paepalanthus stannardii Giul. \& L.R.Parra, Paepalanthus tortilis (Bong.) Mart, Comanthera giuliettiae L.R.Parra, Syngonanthus gracilis (Bong.) Ruhland, Leiothrix schlechtendalii (Körn.) Ruhland, Leiothrix angustifolia (Körn.) Ruhland, Paepalanthus obtusifolius (Steud.) Körn. e Leiothrix distichoclada Herzog. Todas as espécies têm ocorrência na Bahia em vegetação de cerrado ou campo rupestre. Várias amostras não puderam ainda ser identificadas até o nível de espécie, e podem conter novas novas espécies para a região. Várias amostras estavam sem identificação ou identificadas com nomes que não são mais aceitos. Das 72 espécies encontradas na região, 14 estão incluídas na categoria ameaçadas de extinção ou são consideradas espécies raras: Actinocephalus herzogii (Moldenke) Sano, Comanthera curralensis (Moldenke) L.R.Parra \& Giul., Leiothrix distichoclada Herzog, Paepalanthus albo-tomentosus Herzog, Paepalanthus almasensis Moldenke, Paepalanthus barbulatus Herzog, Paepalanthus cinereus Giul. \& L.R.Parra, Paepalanthus contasensis Moldenke, Paepalanthus harleyi Moldenke, Paepalanthus inopinatus Moldenke, Paepalanthus luetzelburgii Herzog, Paepalanthus obtusifolius (Steud.) Körn, Paepalanthus pulvinatus N.E.Br., Paepalanthus stannardii Giul. \& L.R.Parra. 

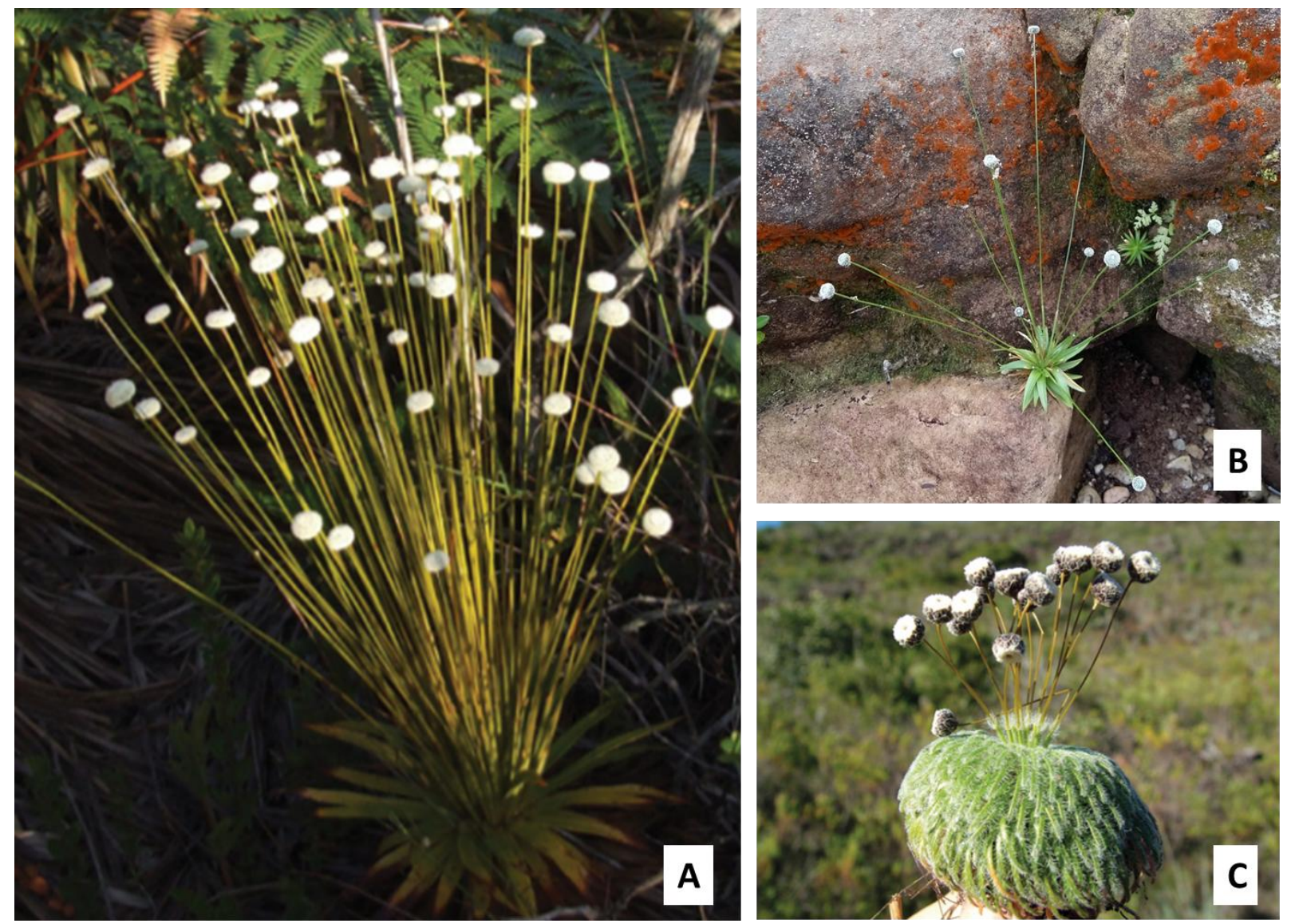

Figura 1. Algumas espécies de Paepalanthus ameaçadas de extinção. A. Paepalanthus barbulatus Herzog. Espécie endêmica da Chapada Diamantina. B. Paepalanthus obtusifolius (Steud.) Körn., espécie endêmica da Bahia. C. Paepalanthus pulvinatus N.E.Br., espécie endêmica da Chapada Diamantina. [Fotos: Marcelo Trovó (A,C), Ariadne Sampaio (B)].

\section{CONCLUSÃO}

A partir dos resultados desse trabalho, principalmente a lista das espécies de Eriocaulaceae ocorrentes nessa área, se percebe que há grande possibilidade de novas ocorrências e novas espécies do grupo na região da Chapada e que o semiárido possui uma riqueza florística ainda menos conhecida do que o necessário e que precisa ser investigada para complementação dos dados nos bancos de dados virtuais de acesso livre.

Foram encontrados ao total 72 espécies de Eriocaulaceae ocorrendo na área da Chapada Diamantina, das 98 relatadas para Bahia. Ainda existem áreas com poucas coletas e poucos estudos, o que justifica a manutenção dos estudos na região.

\section{REFERÊNCIAS}

Giulietti, A.M., Pirani, J.R., (1988). Patterns of geographic distribution of some plant species from the Espinhaço Range, Minas Gerais and Bahia, Brazil, In: Heyer, W.R., Vanzolini, P.E. 
(Eds.), Proceedings of a workshop on Neotropical Distribution Patterns, Academia Brasileira de Ciências, Rio de Janeiro, pp. 39-69.

Giulietti, A.M. \& Hensold, N. (1990) Padrões de distribuição geográfica dos gêneros de Eriocaulaceae. Acta Botanica Brasilica 4: 133-158.

Giulietti, A.M.; Wanderley, M.G.L.; Longhi-Wagner, H.M.; Pirani, J.R. \& Parra, L.R. (1996). Estudos em "sempre-vivas": taxonomia com ênfase nas espécies de Minas Gerais, Brasil. Acta Botanica Brasilica 10: 329-377.

Giulietti, A. M.; Pirani, J. R.; Harley, R. M. (1997) Espinhaço Range Region, Eastern Brazil. In Centres of plant diversity. A guide and strategy for their conservation. (S.D. Davis, V.H. Heywood, O Herrera-Macbryde, J. Villa-Lobos \& A.C Hamilton, eds.). v.3. The Americas, IUCN Publication, Cambridge. p.397-404.

Giulietti, A.M.; Rapini, A.; Andrade, M.J.G.; Queiroz, L.P. \& Silva, J.M.C. (2009). Plantas raras do Brasil. Belo Horizonte, Conservação Internacional.

Giulietti, A.M.; Andrade, M.J.C.; Scatena, V.L.; Trovó, M.; Coan, A.I.; Sano, P.T.; Santos, F.A.R; Borges, R.L.B.; van den Berg, C. (2012) Molecular phylogeny, morphology and their implications for the taxonomy of Eriocaulaceae, Rodriguésia 63(1): 001-019.

Givnish, T.J., Ames, M., McNeal, J.R., McKain, M.R.; Steele, P.R.; Pamphilis, C.W.; Graham, S. W.; Pires, J. C.; Stevenson, D. W.; Zomlefer, W. B.; Briggs, B.G.; Duvall, M. R.; Moore, M. J.; Heaney, J. M.; Soltis, D. E.; Soltis, P. S.; Thiele, K. \& Leebens-Mac, J. H. (2010) Assembling the Tree of the Monocotyledons: Plastome Sequence Phylogeny and Evolution of Poales. Annals of the Missouri Botanical Garden 97(4):584-616.

Rapini, A. (2008) A flora dos campos rupestres da Cadeia do Espinhaço. Tese de Doutorado Universidade de São Paulo, São Paulo.

SANO, P. T. (1999). Revisão de Actinocephalus (Koern.) Sano -Eriocaulaceae- (1999). Tese de Doutorado, Instituto de Biociências da Universidade de São Paulo, São Paulo.

Sano, P. T.; Trovó, M.; Echternacht, L.; Costa, F. N.; Watanabe, M.T.C.; Giulietti, A. M. (2014). Livro vermelho da flora do Brasil - Plantas raras do Cerrado. Rio de Janeiro: Instituto de Pesquisas Jardim Botânico do Rio de Janeiro.

Sano, P.T.; Giulietti, A.M.; Costa, F.N.; Trovo, M.; Echternacht, L.; Tissot-Squalli, M.L.; Watanabe, M.T.C.; Hensold, N.; Andrino, C.O.; Parra, L.R. (2016) Eriocaulaceae in Lista de Espécies da Flora do Brasil. Jardim Botânico do Rio de Janeiro. Disponivel em: <http://floradobrasil.jbrj.gov.br/jabot/floradobrasil/FB110>. Acesso em: 18 Jan. 2016. 